\title{
Study on Crushing Mechanism of Cone Crusher
}

\author{
Hongjun Y $u^{1, a}$, Qiou Fei ${ }^{1, b}$, Rujie Wang ${ }^{1, c}$,Binglu Fan ${ }^{2, d}$, Yamin Wang ${ }^{2, e}$ andBoqiang Shi ${ }^{2, f}$ \\ ${ }^{1}$ Anshan Iron and Steel Group Mining Co., Ltd. equipment manufacturing branch, China \\ ${ }^{2}$ School of Mechanical Engineering, University of Science and Technology Beijing, China \\ ${ }^{a}$ Yhj1958@163.com, ${ }^{b} 8815587 @ q q . c o m,{ }^{c}$ as_wangrujie@sina.com, ${ }^{d}$ 761387461@qq.com, \\ ${ }^{e}$ 1572309925qq.com, ${ }^{f}$ ustb1007@163.com
}

\section{Keywords: EDEM;single particle crushing model;fracture;abrasion}

\begin{abstract}
The single particle crushing model is studied by EDEM software, and the model is extruded with different speeds and different angles. Using horizontal angle of 0 degrees, 20 degrees, 30 degrees, 45 degrees, 60 degrees, 70 degrees plate to squeeze the single particle model, the results show that the ore material is more easily broken down under the complicated stress of tensile shear, when the angle of the plate is 45 degrees, the board change can come to the conclusion that the impact crushing can make the material more easily broken.Simulation of lining wear process, the simulation results show that the wear rate of the dynamic cone is varied, and there is a best speed of the eccentric body.
\end{abstract}

\section{Introduction}

Discrete element theory can describe the mechanical behavior of discontinuous medium such as ore. Therefore, the study of crusher is more focused on the research and simulation analysis of discrete element theory[1]. In addition, when the movable cone liner is worn out, the discharging port will be enlarged, the ore-discharging granularity becomes thicker, and the crushing efficiency is affected. Therefore, the research of the mechanism of breakage includes the study of the wear mechanism of the dynamic cone liner.

\section{Single Particle Extrusion Model}

Particle crushing can be divided into the following three stages: (1) rupture phase:absorption of energy under external forces, resulting in cracks and rupture, the formation of a few large chunks and some broken particles; (2) crushing phase:several blocks continue to be crushed into small pieces or small particles, the gap between the various blocks is more, the energy consumption is not very high;(3) compaction phase: block materials continue to be crushed and smaller fragments and small particles are compacted, due to fine particles filled with the gap between the particles, the interface between the particles increased, the pressure on the brittle material increased dramatically[2].

The particle fragmentation is simulated from the angle of extrusion and the extrusion speed. Through the EDEM software,we can observe the position of crack, the direction of crack propagation and the number of broken bonds in the process of particle crushing, and deduce the crushing force of particles, and calculate the energy consumed in the process of crushing.

\subsection{Effect of Squeezing Angle on Particle Breakage}

In this paper, the single particle model is simulated by changing the angle of the plate to observe the crushing state of the particles. The single particle model was squeezed using a pressure plate with horizontal angles of $0^{\circ}$, 
20 $30^{\circ}, 45^{\circ}, 60^{\circ}$ and $70^{\circ}$, respectively[3].The single particle crushing model is shown in fig. 1 .

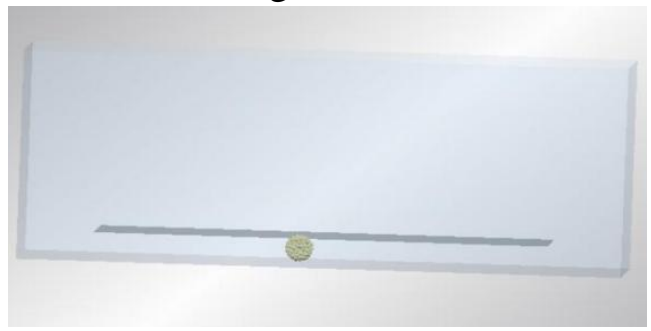

Fig. 1 single particle crushing model

Fig. 2 shows the crushing process of the single particle model when the platen angle is $20^{\circ}$ and the velocity is $0.001 \mathrm{~m} / \mathrm{s}$. The ball particles are hidden and the bonding bond between the particles is preserved[4]. The color of the bond key represents the size of the force between the particles, the blue is the smallest, the green is the second, the red force is the biggest.

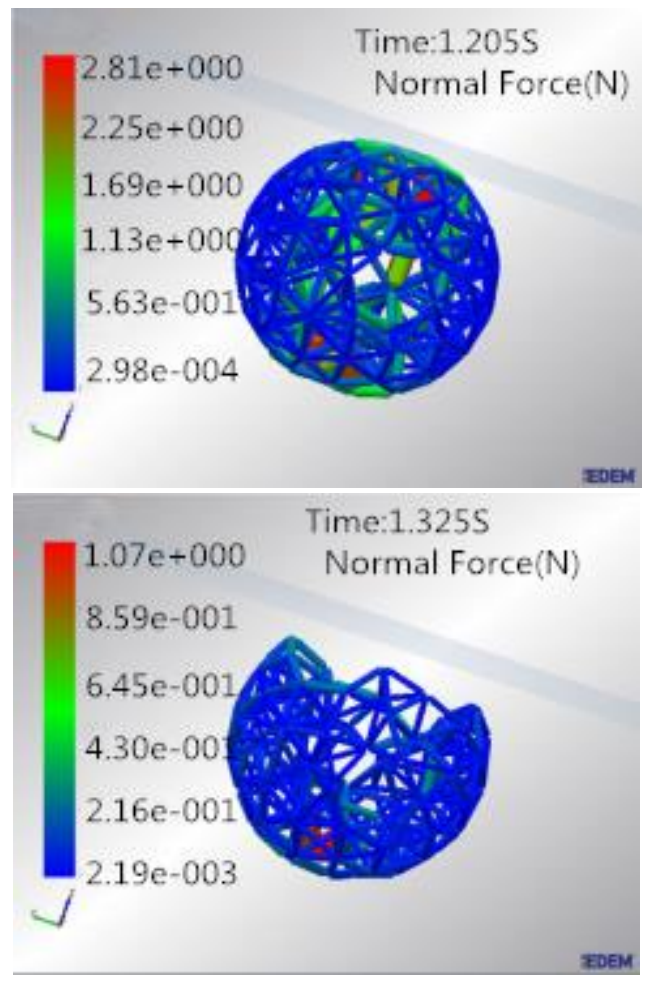

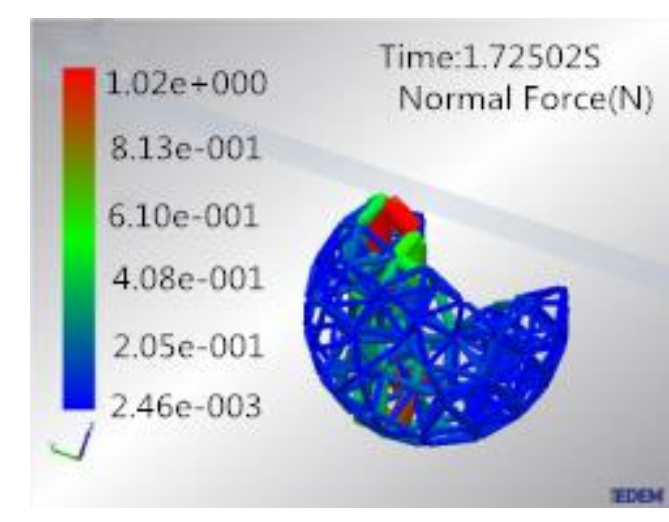

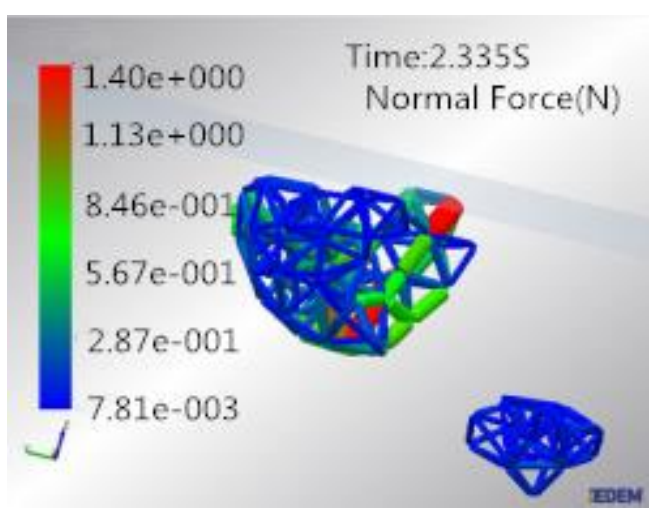

Fig. 2 single particle model crushing process

It can be seen from the analysis, single particles in the process of extrusion, in contact with the pressure plate and the location of the box is the largest. Before the destruction of the particles, the position of the damage will be very strong, but the particles in the rupture after the force is sharply reduced. In the process of the crushing, there is a single particle shedding, which will also produce bulk material particles, the bulk of the material continues to rupture, with the size of the broken material smaller, its plastic characteristics gradually increased, brittle characteristics gradually reduced, materials more difficult to break.

Using the graph generation function in the EDEM post processing function, the resulting line of the pressure plate during the extrusion of the single pellet is generated and the maximum crushing force at different angles is recorded, as shown in table I: 
Table I comparison of crushing forces at different angles

\begin{tabular}{llllllll}
\hline & Platen angle & 0 & 20 & 30 & 45 & 60 & 70 \\
\hline Maximum & Maximum crushing force F(N) & 22.3 & 19.4 & 17.2 & 16.4 & 18.4 & 20.035 \\
\hline
\end{tabular}

From the crushing forces at different angles in table $\mathrm{I}$, the following conclusions can be drawn: When $\theta=45^{\circ}$, the maximum crushing load of limestone material is the smallest, that is, the optimal angle of tensile shear is $45^{\circ}$.

\subsection{Effect of Extrusion Speed on Particle Breakage}

The static pressure of some irregular shape particles of glass balls and other materials and the impact crushing experiments that: (1) The crushing probability of the crushing of material particles is related to the particle size. (2) compared with the static crushing, the impact crushing can break the material with a small energy density[5].

In this paper, the effect of extrusion speed on the crushing force and the breaking energy of single particles was studied by changing the speed of the platen. The graph below shows the crushing force change curves when the extrusion speed is $0.001 \mathrm{~m} / \mathrm{s}$ and $0.1 \mathrm{~m} / \mathrm{s}$, respectively.

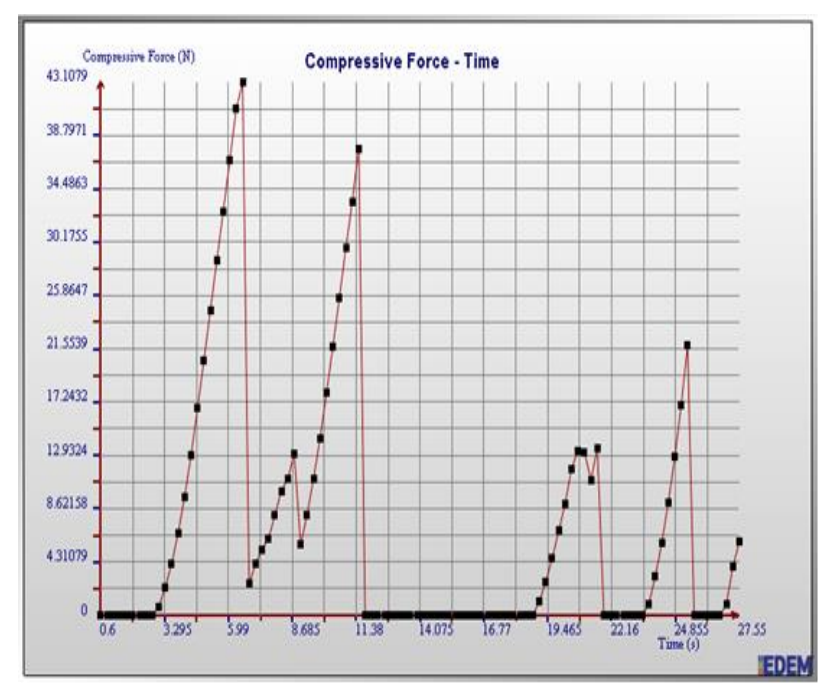

$\mathrm{V}=0.001 \mathrm{~m} / \mathrm{s}$

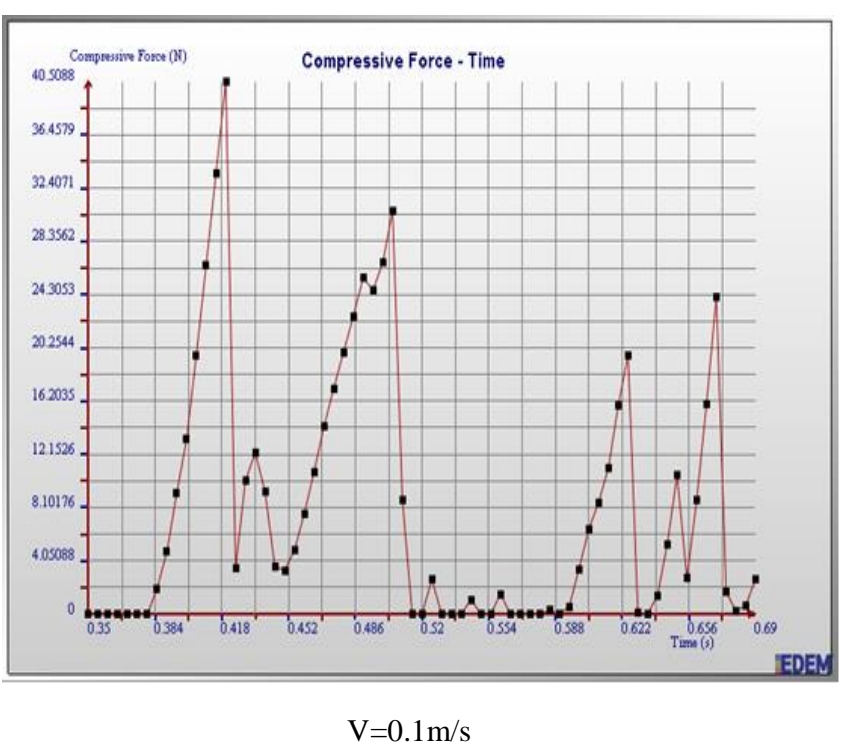

Fig. 3 curve of crushing force at different compression velocities

According to the simulation results, single particles are more easily broken at high impact speeds. Since EDEM can not directly derive the size of the energy after the post-processing, the data of the crushing force is derived and the energy consumed by the particles at the two speeds is obtained by integrating. It can be concluded that the impact of impact crushing can make the material more easily broken, but it consumes more energy than static crushing.

In this section, the single particle model is extruded from different angles at different platen velocities. The results show that under the complex stress of tensile shear, when the angle of the platen is $45^{\circ}$, the ore material is more easily broken, and the impact crushing can make the material more easily broken, but it consumes more energy than the hydrostatic crushing.

\section{Effect of Dynamic Cone Speed on Wear}

The rotational speed of the moving cone is the speed of the eccentric body, which is an important parameter of the crusher. It also has a great influence on the wear of the movable cone liner[6]. 


\subsection{Create a Three-dimensional Model of Cone Crusher}

The software provides a three-dimensional model import function. Therefore, you can use 3D modeling software to model, and then import the model into EDEM. The model of the cone crusher used in the simulation is established by Proe. The figure below shows the three-dimensional solid modeling of the movable cone liner and the fixed cone liner. According to the above-mentioned precession angle, the width of the ore mouth, the minimum size of the discharging port, etc., the geometrical constraint conditions are added in the three-dimensional software to complete the assembling of the crusher.

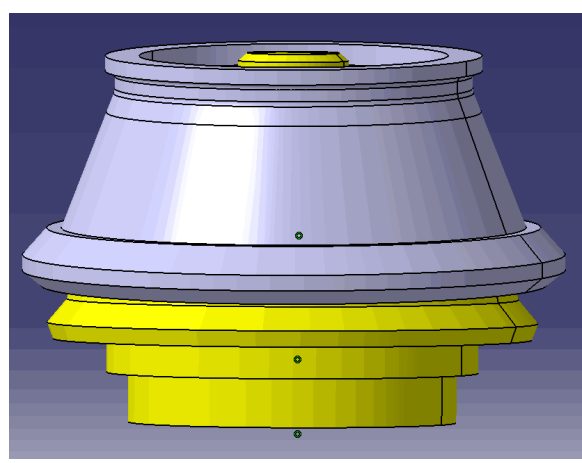

Fig. 4 simplified model of cone crusher

\subsection{Create a Simulation Model}

\section{1) Set the Global Parameters}

The contact model between ore and ore is set to Hertz-Mindlin with bonding model, and the contact model between ore and liner is set to Hertz-Mindlin with Archard Wear model.

\section{2) Archard Wear Contact Model}

The wear constant in the Archard wear model is set to $\mathrm{K}=8.7 \times 10$.

\section{3) Material Parameters}

The material parameters of the model are Materials, the material of the ore material is selected limestone, and the material named Rock, the movable cone liner and the taper liner is named as Steel.By looking up Guo Nianqin's paper data, we can see the material parameters. The establishment of the Fraction to be replaced pellets, set the ball radius of $5 \mathrm{~mm}$, contact radius of $5.5 \mathrm{~mm}$. Set the ball radius of 100 and the contact radius is unchanged. Then, select the material for the particle and the rock. Finally, we choose to automatically calculate the properties of the two particles.

\section{4) Introduce the Cone Crusher Model}

The three-dimensional model of cone crusher built in ProE is imported .Temporarily define dynamic cone liner around Crusher Spindle center line of the Revolution speed is $300 \mathrm{r} / \mathrm{min}$, around its center line rotation speed is $10 \mathrm{r} / \mathrm{min}$, opposite to the direction of the revolution movement.

\section{5) Create a Particle Factory}

The creation of granular factories need to choose the type of granular plant, including dynamic and static two[7]. The first particle plant is used to produce the real particles, the type of particle is selected as static, the type of particles is Whole, the total number is 60, including the initial speed and angular velocity is zero, the particle size is fixed (ratio of 1).The particle location and particle orientation are randomly selected.

Now you can run the simulation. With the free whereabouts of the substituted crushed material under the action of gravity, a certain number of crushing materials will fall into the crushing chamber between the crushing wall and the rolling mortar wall[8].The eccentric sleeve is driven by a swing motion, and the crushing force exerted on the ore is crushed and the ore is broken.

\subsection{Wear Tendency of Dynamic Cone Liner}

The following table is the variation curve of the maximum wear of the movable cone liner during the same crushing time when the eccentric body rotational speed is different. changes with the time curve. 
Table II comparison of the wear of the liner under different eccentric body speed

\begin{tabular}{cccc}
\hline Eccentric b & body speed n $(\mathrm{r} / \mathrm{min})$ & Maximum wear $\omega(\mathrm{mm})$ & Average wear rate v \\
\hline 250 & $5.05348 \times 10^{-4}$ & $1.011 \times 10^{-4}$ \\
300 & $5.55354 \times 10^{-4}$ & $1.111 \times 10^{-4}$ \\
350 & $4.60987 \times 10^{-4}$ & $0.922 \times 10^{-4}$ \\
400 & $9.73787 \times 10^{-4}$ & $1.948 \times 10^{-4}$ \\
\hline
\end{tabular}

The simulation results show that the wear rate of the dynamic cone is variable, and there is an optimum speed of eccentric body. For this simulation, the optimum rotational speed is 350r / min.

\section{Conclusion}

The single particle model was extruded from different angles with different platen velocities. The results show that when the angle of the platen is $45^{\circ}$, the ore material is more easily broken and the impact crushing can make the material more easily broken. The wear rate of the dynamic cone is changeable, and there is an optimum rotational speed of the eccentric body, which can minimize the wear of the liner.

\section{References}

[1]Nianqin Guo, Haolong Zheng et al. 2015.Experimental study on the simulation of laminated crushing model based on EDEM cone cusher [J]. Mining Equipment, 43 (4): 61-66.

[2]Xiaoliang Chang. 2012.Discrete element numerical simulation analysis of grinding of ball mill experiment machine[D]. Kunming University of Science and Technology.

[3]Binglu Fan. 2015.Study on the mechanism of cone crusher based on discrete element [D] .Beijing University of Science and Technology .

[4]Jianguo Li.2016.Research on crushing process of cone crusher based on EDEM [D]. Beijing University of Science and Technology. [5]Haiyan Li. 2011. Simulation of performance parameters of vertical screw conveyor based on EDEM[D]. Taiyuan University of Science and Technology .
[6]Refahi A, Aghazadeh M J, Rezai B. 2010 .Discrete element modeling for predicting breakage behavior and fracture energy of a single paticle in a jaw crusher [J]. International Journal of Mineral Processing, (2):83-91.

[7]Xiufang Wang. 2011.Evaluation of mechanical properties of cement materials and concept of small energy crushing[D]. China Building Materials Science Research Institute . [8]Bo Zhou, Runqiu Huang, Huabin Wang, et al.2014.Study on the evolvement law of sandy soil fragmentation based on discrete element method[J]. Rock and Soil Mechanics, 35 (9): 2709-2716. 